\title{
Secreted Probiotic Factors Ameliorate Enteropathogenic Infection in Zinc-Deficient Human Caco-2 and T84 Cell Lines
}

\author{
ILSE J. BROEKAERT, N. NANDA NANTHAKUMAR, AND W. ALLAN WALKER
}

\begin{abstract}
Department of Pediatrics [I.J.B., N.N.N., W.A.W.], Mucosal Immunology and Developmental Gastroenterology Laboratories, Massachusetts General Hospital for Children, Harvard Medical School, Boston, Massachusetts 02215; Dr. von Hauner Children's Hospital [I.J.B.], University of Munich, 80337 Munich, Germany
\end{abstract}

\begin{abstract}
Zinc deficiency enhances infectious diarrhea whereas probiotics may inhibit pathogen enterocyte invasion. The effect of probiotics on zinc-deficient versus normal human intestinal epithelium (Caco-2 and T-84) with regard to invasion and subsequent inflammatory response by Salmonella typhimurium was determined. Cells were infected with pathogens and preincubated with media conditioned by several lactobacilli or Bifidobacterium bifidum 12 . Pathogen invasion was quantified, inflammation was determined by IL- 8 secretion, and MAP kinase activation in the epithelium was analyzed. Probiotic inhibiting factors were partially characterized based on physicochemical properties. Zinc deficiency allowed for greater pathogen invasion and enhanced IL-8 secretion. Probiotic conditioned media reduced activation of proinflammatory signaling via the ERK and p38 pathway. Probiotic factors reverse increased susceptibility of zinc-deficient enterocytes to S. typhimurium invasion, suggesting an additive protective effect of probiotics in zinc deficiency. Probiotic conditioned media but not bacteria inhibited pathogen invasion and IL-8 production in zinc deficient enterocytes. Probiotic inhibitory factors are stable to treatment with proteases, deoxyribonucleases (DNAses), ribonucleases (RNAse), strong acid, and heat. (Pediatr Res 62: 139-144, 2007)
\end{abstract}

$\mathrm{Z}$ inc deficiency is a common problem in both developing and developed countries. One third of the children in sub-Saharan Africa and South East Asia are severely zinc deficient (1) due to severe malnutrition. Mild to moderate zinc deficiency is prevalent in the aging population of industrialized countries (1). Deficiency can develop iatrogenically, e.g. in premature infants or the elderly with prolonged total parenteral nutrition and when medicated with diuretics (2). Mild deficiency often goes undetected but moderate to severe zinc deficiency leads to fulminant diarrhea, growth retardation, immune deficiency, desquamating eczema, hypogonadism, and lethargy (3). Infectious diarrhea is associated with zinc depletion (4) and zinc supplementation has been shown to ameliorate diarrhea (5). Thus, zinc sufficiency appears to be an important factor in the prevention and management of infectious diarrhea.

Received November 17, 2006; accepted March 23, 2007.

Correspondence: W. Allan Walker, M.D., Mucosal Immunology and Developmental Gastroenterology Laboratories, MA General Hospital, 114 16th Street (114-3503), Charlestown, MA 02129; e-mail: wwalker@partners.org

This review was supported by funding from the National Institutes of Health [(PO1 DK33506, P30 DK40561, R37 HD12437 (W.A.W.), ROI DK70260 (W.A.W., N.N.N.)], by a Fulbright Scholarship from the United States Department of State (I.J.B.), and by a research fellowship from Mead Johnson Nutritionals (I.J.B.).
Probiotics are health-promoting colonizing bacteria with strain-specific effects on the mucosal barrier and intestinal host defenses (6) by playing a role in inhibiting colonization by other intestinal microflora (7). Probiotics have been used to prevent and treat infectious diarrhea in infancy (8). A metaanalysis suggested that they could reduce the duration and severity of diarrhea (9).

Since both treatments have similar effects in the management of infectious diarrhea, we hypothesized that probiotics have a beneficial effect on pathogen infection in zinc deficient human intestinal cells. They even can overcome the host defense defect caused by zinc deficiency.

\section{METHODS}

Chemicals. The following chemicals were used: BSA (Celliance, Atlanta, GA); calcium carbonate, copper sulfate, L-cysteine hydrochloride, lead (II) nitrate, and magnesium chloride hexahydrate (Sigma Chemical Co., St. Louis, $\mathrm{MO}$ ); nonessential amino acids, penicillin and gentamicin (Invitrogen, Rockville, MD); fetal bovine serum (FBS; Atlanta Biologicals, Lawrenceville, GA); tissue culture plastics (Fisher Scientific, Lincoln Park, NY); and IL-8 ELISA components (Pierce, Rockford, IL).

Cell lines. Adult colonic adenocarcinoma cells (Caco-2 and T84 cells) (ATCC, Manassas, VA) were grown in Dulbecco's modified eagle medium (DMEM) supplemented with 10\% FBS (Caco-2) or in a 1:1 (vol/vol) mixture of DMEM and Ham's F12 media supplemented with 5\% FBS (T84). The media was supplemented with $1 \%$ nonessential amino acids, $50 \mathrm{IU} / \mathrm{mL}$ penicillin, $50 \mu \mathrm{g} / \mathrm{mL}$ streptomycin, and $1 \%$ HEPES buffer. Cells were grown at $37^{\circ} \mathrm{C}$ in $95 \% \mathrm{O}_{2}$ and $5 \% \mathrm{CO}_{2}$.

Bacteria. Strains frozen at $-80^{\circ} \mathrm{C}$ in glycerol were subcultured twice at $37^{\circ} \mathrm{C}$ under aerobic (pathogenic bacteria) or anaerobic (probiotics) (BBL Campy Pak Plus Microaerophilic System, BD Biosciences, Franklin Lakes, NJ) conditions. Bifidobacterium bifidum 12 was kindly provided by Dr. G. Prioult, Massachusetts General Hospital, Charlestown, MA. Lactobacillus acidophilus (ATCC 4356), L. casei (ATCC 393), L. crispatus (ATCC 33197), L. delbrueckii (ATCC 11842), L. fermentum (ATCC 9338), L. paracasei (ATCC 11974), L. plantarum (ATCC 8014), and L. rhamnosus GG (ATCC 53103) were obtained from ATCC. B. bifidum 12 was grown in MRS supplemented with $0.5 \mathrm{~g}$ of L-cysteine/L. Probiotic bacteria were grown at a concentration of $10^{9} \mathrm{cfu} / \mathrm{mL}$. Conditioned media was prepared by centrifugation of probiotic bacteria at $3000 \mathrm{~g}$ for $10 \mathrm{~min}$ and by filtration to eliminate residual bacteria. Purified probiotic bacteria were washed with PBS and centrifuged twice at $3000 \mathrm{~g}$ for $10 \mathrm{~min}$. Pathogenic Salmonella enterica serovar typhimurium (SL1344 or F98) was kindly provided by Drs. B. Cherayil and B. McCormick (Massachusetts General Hospital, Charlestown, MA). Bacterial growth media were obtained from BD Biosciences [MRS for probiotics, Luria Bertani (LB) for S. typhimurium].

Preparation of zinc-deficient cell culture medium. We defined zinc deficiency as levels below $0.05 \mathrm{mg} / \mathrm{L}$ based on experiments of Bao et al. (10), who showed that zinc deficiency $(\leq 0.05 \mathrm{mg} / \mathrm{L})$ leads to increased tumor

Abbreviations: MRS, Mann Rogosa Sharpe; PMA, phorbol 12-myristate 13-acetate 
necrosis factor (TNF)- $\alpha$, IL-1 $\beta$, and IL-8 and decreased IL-2 and interferon (IFN)- $\gamma$ levels in human malignant T-lymphoblasts and monocytemacrophages. Zinc-deficient medium was prepared by chelating zinc from FBS, DMEM, and DMEM/F12 1:1 (vol/vol). DMEM contains $40 \mathrm{ng} / \mathrm{mL}$ zinc, DMEM/F12 1:1 (vol/vol) 92 ng/mL, FBS 2493 ng/mL zinc. Chelating resin (iminodiacetic acid; Sigma Chemical Co.) was treated with $\mathrm{Ca}_{2} \mathrm{CO}_{3}$ acidified with $5 \mathrm{M} \mathrm{HCl}$ and washed with zinc-free water until neutral (10). Twenty-five grams treated resin was added to $500 \mathrm{~mL} \mathrm{FBS}$ and stirred at $4^{\circ} \mathrm{C}$ overnight (up to six times). Complete growth medium was reconstituted by replenishing copper, magnesium, lead, and manganese to bring ions back to original concentration.

Cellular zinc assay. Samples of DMEM, DMEM/F12 1:1 (vol/vol) and FBS were analyzed for ion content (zinc, copper, magnesium, lead, and manganese) before and after chelation. Zinc concentrations were determined by inductively coupled plasma mass spectrometry (ICP-MS) (Varian Instruments, Palo Alto, CA) (kindly performed by Dr. Chitra Amarasiriwardena, Harvard School of Public Health, Boston, MA). For calibration, a bovine liver reference standard (National Institute for Standards and Technology, Gaithersburg, MD) was used. Zinc deficiency was defined as less than $0.05 \mathrm{mg} / \mathrm{L}$ (10). In our experiments, zinc deficient Caco-2 and T84 cells had significantly lower zinc levels $(0.064 \mathrm{mg} / \mathrm{L}$ and $0.064 \mathrm{mg} / \mathrm{L}$, respectively) than cells grown in normal cell culture media $(0.414 \mathrm{mg} / \mathrm{L}$ and $0.381 \mathrm{mg} / \mathrm{L}$, respectively).

Invasion assay and induction of IL-8. Caco- 2 and T84 cells were grown in 24-well tissue culture dishes for one week after reaching confluence. Zinc-deficient cells were cultured in zinc-deficient media 1-wk before invasion assay. Culture media was changed to antibiotic and serum-free media $24 \mathrm{~h}$ before the invasion assay. Cells were infected with $10^{8} \mathrm{~S}$. typhimurium (SL1344 or F98) with or without probiotic conditioned media or purified probiotic bacteria. PBS and MRS media were used as negative controls for the probiotic conditioned media. To find out whether MRS interferes with pathogenic invasion it was compared with PBS as negative control. After $1 \mathrm{~h}$, pathogens were washed away, and cells were incubated in $100 \mu \mathrm{g} / \mathrm{mL}$ gentamicin for $90 \mathrm{~min}$ to kill remaining extracellular pathogens. Cell supernatants were collected for IL-8 measurement. Cells were washed and lysed in sterile water with $1 \%$ Triton $\mathrm{X}-100$. Intracellular bacteria were quantified by plating $100-\mu \mathrm{L}$ aliquots of cell lysates on LB plates. Colony-forming units were quantified after overnight incubation at $37^{\circ} \mathrm{C}$ to estimate pathogen invasion.

Protein determination. Protein concentrations were measured by a bicinchoninic acid protein assay (BCA assay, Pierce) in 96-well microtiter plates. Absorbance was measured at $625 \mathrm{~nm}$ using a spectrophotometer (Spectra Max 190, Molecular Devices, Sunnyvale, CA). The protein concentration was calculated based on a BSA standard curve.

IL-8 measurement by ELISA. IL-8 was measured by a double-sandwich ELISA technique (11). Ninety-six-well, high-binding microtiter plates were coated overnight with mouse anti-human IL-8, blocked with BSA, and incubated at room temperature for 60 min with $50 \mu \mathrm{L}$ of the cell supernatants and standards. Plates were washed between incubation steps. After incubation with mouse anti-human IL-8 detecting antibody, horseradish peroxidaseconjugated streptavidin and 3,3',5,5' -tetramethylbenzidine substrate solution, absorbance was measured at $450 \mathrm{~nm}$ using a spectrophotometer (Spectra Max 190). IL-8 concentrations were determined from a standard curve with purified recombinant human IL-8 and expressed in relation to total cellular protein.

Western blotting. Caco-2 and T84 cells were grown in six-well tissue culture dishes. Cells were treated with pathogens with or without probiotic bacteria and lysed with lysis buffer [10 mM HEPES pH 7.6, $150 \mathrm{mM} \mathrm{NaCl}$, $1.5 \mathrm{mM} \mathrm{MgCl}, 10 \mathrm{mM} \mathrm{KCl}, 1 \mathrm{mM}$ EDTA, 1 mM EGTA, $4 \mathrm{mM} \mathrm{Na}_{3} \mathrm{VO}_{4}, 40$ $\mathrm{mM} \mathrm{NaF}$, protease inhibitor cocktail (Sigma Chemical Co.), phosphatase inhibitor cocktails I and II (Sigma Chemical Co.), Triton-X 100 1\%, SDS $0.5 \%)]$. After estimating protein concentrations by BCA assay, $35 \mu \mathrm{g}$ of denatured cell lysate was fractionated in SDS-PAGE gel (Criterion XT Precast Gel, 4-12\% Bis-Tris, Bio-Rad, Hercules, CA) and transferred onto polyvinylidene fluoride (PVDF) (Bio-Rad) using a semi-dry transfer cell (Transblot SD, Bio-Rad). Membranes were blocked in 5\% nonfat milk in Tris-buffered saline (TBS) plus $0.1 \%$ Tween (TBST) before being probed with primary antibodies [anti-phospho-extracellular signal-regulated kinases (ERK), anti-ERK, anti-phospho-p38 and anti-p38 (Cell Signaling, Beverly, MA)] at a dilution of 1:250 overnight. Phosphorylation of kinases indicates activation. As positive control for phosphorylated ERK, enterocytes were incubated with $100 \mathrm{nM}$ phorbol 12-myristate 13-acetate (PMA) (Sigma Chemical Co.). Membranes were washed thrice with TBST and probed with rabbit anti-mouse IgG linked to horseradish peroxidase (Pierce) as a secondary antibody (1:500 dilution) for $1 \mathrm{~h}$. Membranes were developed with enhanced chemiluminescence detection (Pierce) and intensity of the bands was quantified by densitometry (Applied Biosystems, San Diego, CA) using unsaturated films with NIH Image software (12).
Characterization of probiotic inhibitory factors. Soluble probiotic factors released into culture media were characterized as to physicochemical properties. D-lactic acid was diluted in PBS to $100 \mathrm{mM}$. Heat-killed probiotic bacteria were obtained by boiling probiotic culture for $10 \mathrm{~min}$. The $\mathrm{pH}$ of probiotic-conditioned media was between 4 and 5 maximally. pH changes were induced by adding trichloretic acid to the probiotic conditioned media and by readjusting to either $\mathrm{pH} 4-5$ or 7.4 (pH of PBS and MRS as negative control) before incubating the enterocytes. Trichloretic acid was used to maximize $\mathrm{pH}$ conditions to determine the effect of $\mathrm{pH}$ on the inhibitory factors. Deoxyribonuclease I (Sigma Chemical Co.; 2580 units/mg solid) and II (Sigma Chemical Co.; 2054 units/mg solid), ribonuclease A (Sigma Chemical Co.; 45 units/mg solid), and protease (Sigma Chemical Co.; 0.9 units $/ \mathrm{mg}$ solid) at concentrations of $10 \mu \mathrm{g}$ per $100 \mu \mathrm{L}$ of media were added to conditioned media and adjusted to the $\mathrm{pH}$ of optimal enzyme activity. After 60 min of incubation, enzymes were inactivated. As positive controls, untreated probiotic conditioned media that underwent heat treatment and $\mathrm{pH}$ changes was used.

Statistics. Results are presented as the mean \pm SD. Significant effects in enterocyte invasion and IL-8 secretion were analyzed by $t$ test. A difference with a $p$-value $\leq 0.05$ was considered significant.

\section{RESULTS}

Role of probiotics in pathogen invasion and inflammation in zinc-deficient and -sufficient cells. Invasion of Caco-2 cells with $S$. typhimurium was significantly enhanced ( 3.2 fold) in zinc-deficient versus normal enterocytes (Fig. 1A). IL-8 was expressed at basal levels in Caco-2 cells (Fig. 1B) and T84 cells (results not shown) and was equal in zinc-deficient and
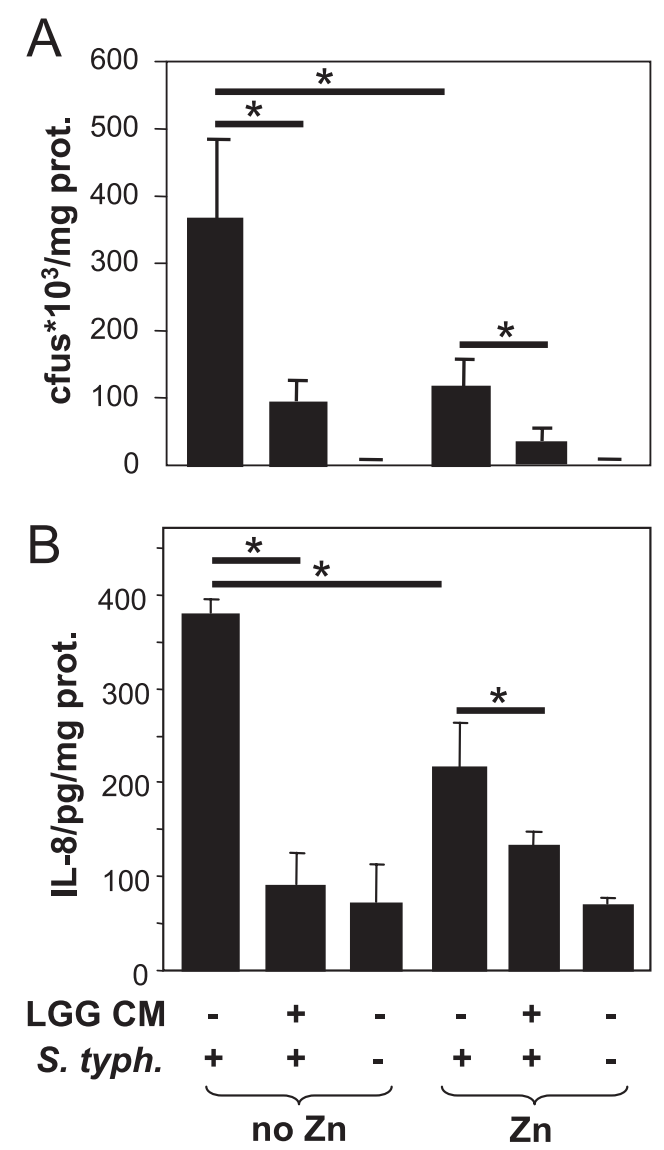

Figure 1. L. rhamnosus $\mathrm{GG}$ conditioned media $(L G G C M)$ inhibits invasion and inflammatory response in zinc deficient Caco-2 cells. S. typhimurium (S. typh.) invasion $(A)$ and IL-8 response $(B)$ of cells grown in zinc deficient (no $\mathrm{Zn}$ ) was compared with those grown in normal medium $(\mathrm{Zn})$. Colony-forming units $(c f u)$ as measure of invasion $(A)$ and IL-8 response $(B)$ to determine inflammatory response corrected for cell concentration in milligrams protein (prot.) were quantified. Significant reduction $(*)$ was defined as $p<0.05$. 
A

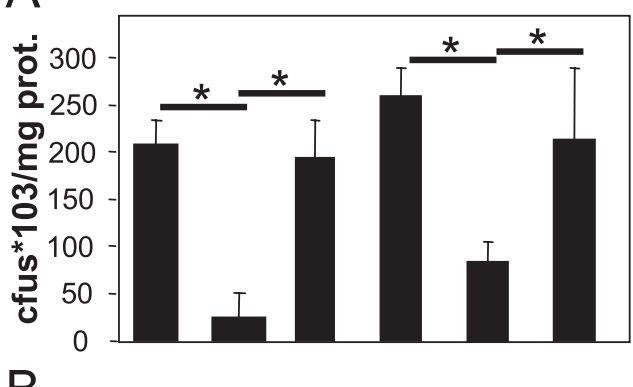

B

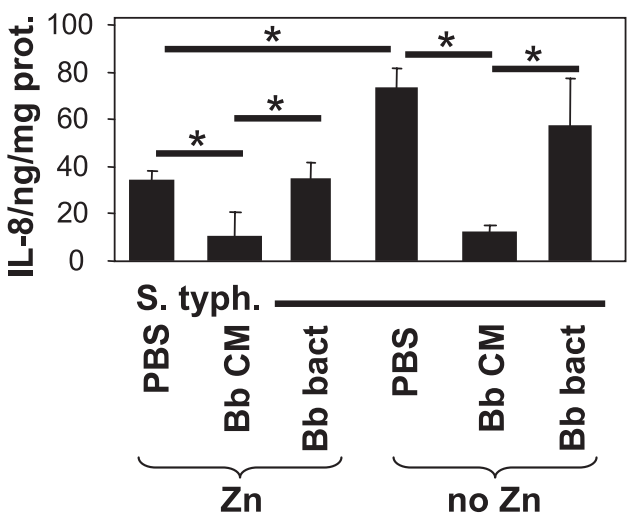

Figure 2. Washed probiotic bacteria do not inhibit $S$. typhimurium invasion and IL-8 secretion in enterocytes. B. bifidum 12 conditioned media $(B b C M)$, purified $B$. bifidum 12 bacteria ( $B b$ bact), and PBS as negative control were tested on Caco-2 cells. Pathogen invasion $(A)$ and IL-8 response $(B)$ were quantified. Significant reduction $(*)$ was defined as $p<0.05$.

normal Caco-2 cells at basal state (Fig. 1B). Upon S. typhimurium infection, IL-8 secretion was induced 5.3-fold in zinc-deficient and 3.2-fold in normal Caco-2 cells (Fig. 1B). IL-8 secretion was significantly enhanced in zinc deficient Caco-2 cells by 1.8 (Fig. $1 B$ ) and by 2.2 (Fig. 2) compared with zinc-sufficient Caco-2 cells.

Probiotic conditioned media harvested at increasing optical densities representing different bacterial concentrations inhibited pathogen invasion in a concentration dependent manner (Fig. 3). L. rhamnosus GG conditioned media significantly inhibited $S$. typhimurium invasion by $75 \%$ in zinc-deficient compared with normal (64\%) Caco-2 cells (Fig. 1A). The probiotic inhibitory effect was enhanced in zinc-deficient compared with zinc-sufficient T84 cells (Fig. 4) in case of L. rhamnosus GG (96 versus 78\%), B. bifidum (88 versus $67 \%)$, L. plantarum (89 versus $75 \%$ ) and slightly enhanced in the case of $L$. casei ( 78 versus $76 \%$ ) and L. paracasei (76 versus $72 \%)$.

L. rhamnosus GG conditioned media significantly reduces $S$. typhimurium induced inflammation in Caco-2 cells (Fig. $1 B)$. The inhibitory effect is enhanced in zinc-deficient $(76 \%)$ compared with zinc-sufficient (39\%) enterocytes (Fig. 1B). $B$. bifidum conditioned media reduced IL- 8 secretion by $82 \%$ in zinc-deficient compared with normal $(71 \%)$ Caco-2 cells (Fig. 2).

PBS and MRS were used as negative controls for the probiotic conditioned media. It could be shown that the inhibitory effect of probiotic conditioned media is not due to potential factors in the bacterial growth media (Fig. 3, Fig. 4).
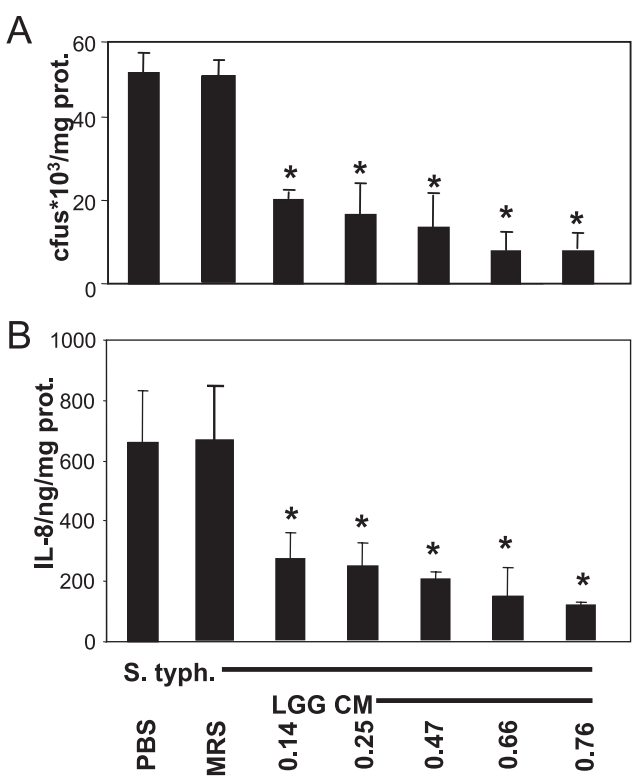

Figure 3. L. rhamnosus GG conditioned medium harvested at increasing optical densities inhibits $S$. typhimurium invasion (A) and IL-8 secretion $(B)$ in zinc sufficient T84 cells in a concentration-dependent manner compared with PBS and MRS as negative controls. The inhibitory effect of L. rhamnosus GG conditioned media is not due to potential factors in the bacterial growth media. LGG CM L. rhamnosus GG conditioned medium, OD. *p $<$ 0.05 .

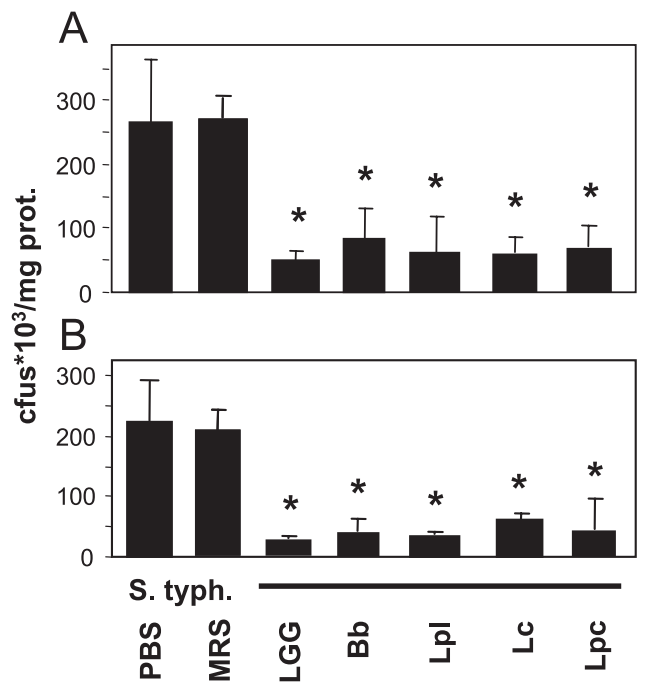

Figure 4. Probiotic conditioned media inhibit $S$. typhimurium invasion in zinc-deficient $(A)$ and -sufficient $(B)$ enterocytes. T84 cells were infected with $S$. typhimurium in the presence of conditioned media of different lactobacilli $[L$. rhamnosus GG $(L G G)$, L. plantarum $(L p)$, L. casei $(L c)$, or L. paracasei $(L p c)]$, B. bifidum $12(B b)$, PBS, and MRS media (MRS). MRS media does not possess inhibitory properties toward pathogenic invasion of zinc-deficient and -sufficient Caco-2 cells whereas different probiotic conditioned media are significantly inhibitory. Significant reduction $(*)$ was defined as $p<0.05$.

Signal transduction in zinc-deficient enterocytes treated with pathogenic bacteria and probiotics. To understand underlying mechanisms of increased invasion and enhanced IL-8 secretion mitogen-activated protein kinase (MAPK) pathway activation was examined. p38 and c-Jun-N-terminal kinase (JNK) and ERK are part of the MAPK pathway. We looked at the activation by phosphorylation of ERK and p38 upon infection. There is some baseline activation of ERK in zinc- 
Zn-deficient Caco-2

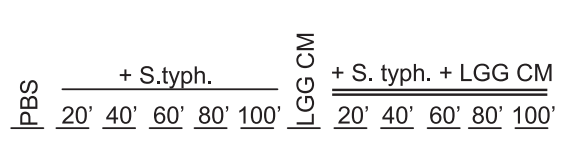

p-ERK

ERK

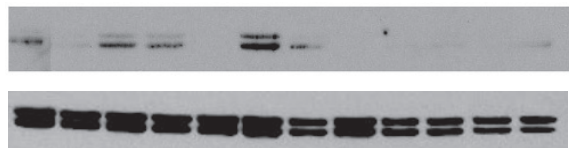

Zn-sufficient Caco-2
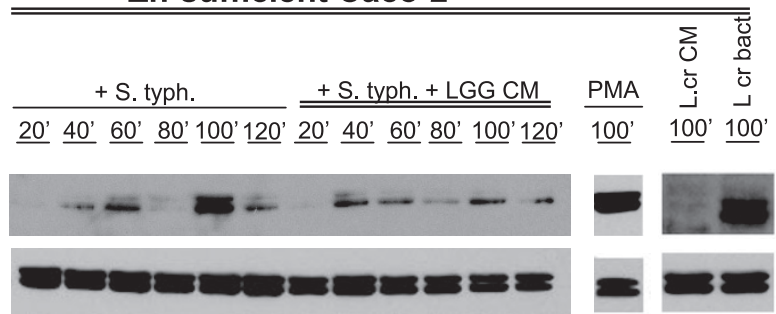

Figure 5. ERK-activation is reduced in infected zinc-deficient and normal Caco-2 cells by probiotics. Infected Caco-2 cells grown in zinc-deficient (no Zn) or normal $(\mathrm{Zn})$ medium and infected with S. typhimurium (S. typh.) in the presence or absence of $L$. rhamnosus GG $(L G G C M)$ were lysed at different time intervals $(20,40,60,80,100$, and $120 \mathrm{~min})$. Activated ERK (p-ERK) was quantified via Western blotting and compared with total ERK and the strong ERK-activator PMA at a dose of $100 \mu \mathrm{M}$. To prove that probiotic conditioned media and not washed $L$. crispatus inhibits ERK activation Caco-2 cells were incubated with S. typhimurium in the presence of L. crispatus CM and washed bacteria. As negative controls PBS and LGG CM in zinc-deficient and sufficient Caco-2 (results not shown) cells were used without pathogen.

deficient and normal enterocytes (Fig. 5). As a positive control the strong ERK activator PMA was used (Fig. 5). ERK activation upon infection of Caco-2 cells with S. typhimurium is time-dependent (Fig. 5) and biphasic with peaks at 40-60 and 100 min postinfection (Fig. 5). However, it does not reach the level of PMA-induced phosphorylated ERK (Fig. 5). The concentration of phosphorylated ERK is reduced in zincdeficient versus zinc-sufficient Caco-2 cells (Fig. 5). There is some baseline activation of p38 in zinc-deficient enterocytes but not in normal enterocytes (Fig. 6). S. typhimurium causes a significant increase in activated p38 after $2 \mathrm{~h}$ of incubation of the enterocytes. p38 activation is reduced in zinc-deficient cells compared with normal cells (Fig. 6).

L. rhamnosus GG conditioned media reduces the concentration of phosphorylated ERK in Caco-2 cells upon S. typhimurium infection (Fig. 5). In zinc-deficient enterocytes probiotic conditioned media totally abolished ERK activation whereas in normal enterocytes some phosphorylated ERK is still present (Fig. 5). L. rhamnosus GG conditioned media reduces p38 activation in both zinc-deficient and normal cells infected with S. typhimurium (Fig. 6). In zinc-deficient cells p38 activation is totally abolished (Fig. 6).

Effect of probiotic conditioned media versus washed probiotic bacteria. Different purified probiotic bacteria removed from conditioned media were significantly less inhibitory in

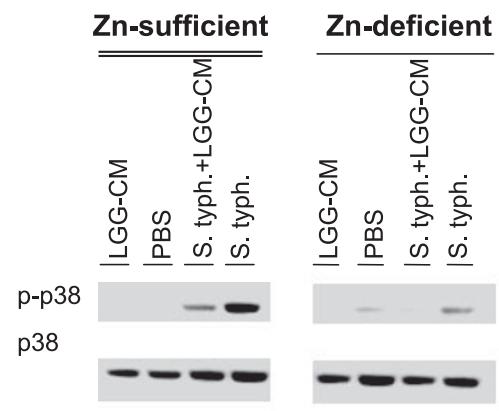

Figure 6. L. rhamnosus $\mathrm{GG}$ conditioned medium inhibits $\mathrm{p} 38$ activation upon S. typhimurium invasion. Caco-2 cells grown in normal or zinc-deficient media, infected with S. typhimurium (S. typh.) in the presence of L. rhamnosus GG conditioned media ( $L G G-C M)$ or MRS, were lysed and incubated with anti-phospho-p38 (p-p38) and total anti-p38 (p38). Uninfected Caco-2 cells treated with MRS and LGG-CM were used as negative controls. Activation of p38 was quantified via Western blotting and compared with total p38. pathogen invasion. Purified B. bifidum had only a minor influence in zinc-deficient Caco-2 cells and did not inhibit IL-8 secretion in normal Caco-2 cells (Fig. 2).

Purified probiotic bacteria such as L. crispatus were not active in the inhibition of ERK-activation (Fig. 2).

Preliminary characterization of $L G G$ and $L$. plantarum secreted molecules on invasion and inflammation. All probiotic-conditioned media showed significant inhibitory effects in inhibiting pathogen invasion and IL-8 secretion whereas purified probiotic bacteria are only minimally active. $L$. $r h$ amnosus GG and L. plantarum products were analyzed to partially characterize inhibitory factors. Inhibitory factors in L. rhamnosus GG (results not shown) and L. plantarum (Fig. 7) conditioned media were shown to be resistant to treatment with proteases, deoxyribonucleases and ribonuclease separately. Heat and $\mathrm{pH}$ changes (Fig. 7) did not affect inhibitory activity of probiotic-conditioned media. The inhibitory activity was not lost if the probiotic conditioned media was adjusted to $\mathrm{pH} 7.4$ (results not shown). D-lactic acid at a concentration of $100 \mathrm{mM}$ partially inhibits S. typhimurium invasion in Caco-2 cells compared with L. rhamnosus GG conditioned media (55\% versus $92 \%$ ) proving that the effect of L. rhamnosus GG conditioned media is both due to lactic acid and other inhibitory factors (results not shown). Accordingly, partial characterization of secreted inhibitory factors from $L$. rhamnosus GG and L. plantarum suggests that these factors are neither a protein nor nucleic acid. Further studies are underway to find out if the inhibitory factors are polysaccharides or fatty acids.

\section{DISCUSSION}

Zinc deficiency is widely prevalent in developed and developing countries and zinc may be an important adjunct in prevention or treatment of diarrhea. Normal serum zinc levels lie between 0.6 and $1.1 \mathrm{mg} / \mathrm{L}$ and in zinc deficiency supplementation is achieved with roughly $1 \mathrm{mg} / \mathrm{kg} / \mathrm{d}$. Probiotics are beneficial in prevention and treatment of infectious gastrointestinal diseases. Previous studies have shown positive effects of zinc and probiotics on intestinal inflammation $(13,14)$. Diarrhea causes zinc depletion in enterocytes with disadvantageous immune effects. We show for the first time that enteropathogenic invasion and inflammatory response are en- 


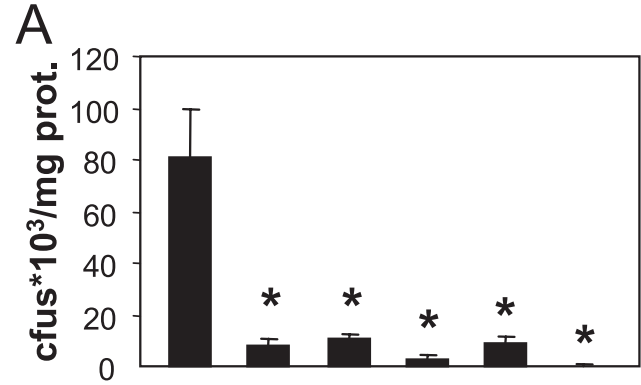

B

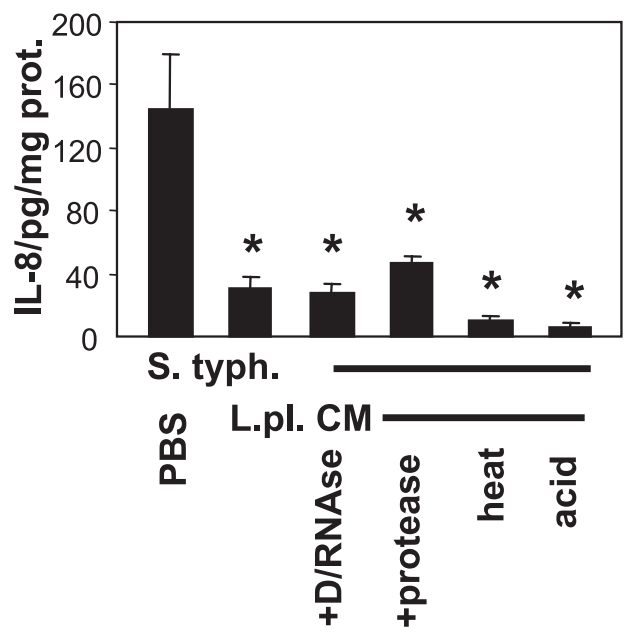

Figure 7. L. plantarum conditioned medium was treated with enzymes, heat, and acid to characterize the inhibitory factor(s) and tested for efficiency in $S$. typhimurium infected T84 cells. S. typh., S. typhimurium; L.pl. CM, L. plantarum conditioned medium; D/RNAse, deoxyribonucleases and ribonucleases; $c f u$, colony-forming units; prot., protein. ${ }^{*} p<0.05$.

hanced in zinc-deficient enterocytes. Our studies also provide the first in vitro evidence that probiotics can overcome increased susceptibility of zinc-deficient cells to enteropathogenic bacteria.

Media conditioned by different lactobacilli or B. bifidum 12 significantly inhibited pathogen invasion and IL-8 secretion in infected zinc-deficient enterocytes. Enhanced IL-8 secretion in zinc-deficient enterocytes could be due to either an increased number of intracellular pathogenic bacteria or a direct "overreaction" of the inflammatory pathway in zinc deficiency. Previous studies (15) have shown that pathogen infection leads to stimulation of the MAP kinase pathway leading to nuclear factor (NF)- $\kappa \mathrm{B}$ activation, transcription, and secretion of IL-8. There was no inhibition of IL- 8 by purified bacteria and probiotic conditioned media did not induce IL- 8 secretion alone at a concentration of approximately $10^{9} \mathrm{cfu} / \mathrm{mL}$. The zinc content in probiotic-conditioned media $(1169 \mathrm{ng} / \mathrm{mL})$ is similar to MRS media (1122 ng/mL) and MRS alone did not inhibit IL-8 secretion. Therefore, the protective probiotic effect in zinc deficiency is not conferred by the zinc content of the conditioned media. Mengheri et al. (13) have shown that treatment of zinc-deficient mice with Bifidobacterium animalis protects the gut from alterations induced by zinc deficiency and that zinc supplementation alone does not confer beneficial effects on the intestine. They propose that probiotics are able to modulate the immune response directly by altering cytokine production in zinc-deficient enterocytes (16). We made the observation that the probiotic effect was enhanced in zincdeficient compared with sufficient enterocytes and propose a synergistic effect of probiotics and zinc.

To understand the underlying mechanism the activation of the MAP kinase pathways were examined. Our observations suggest that the increase in IL-8 upon pathogen infection is mediated by the same signaling pathway in zinc deficient and sufficient enterocytes. Our results showed that $S$. typhimurium increases the activation of both the ERK and p38 pathway. We showed that activation of ERK is reduced in zinc-deficient enterocytes. Azriel-Tamir et al. (17) described how zinc specifically activates ERK in HT-29 cells, which entails the activation of inositol triphosphate (IP3) causing a rise in intracellular calcium. Zinc activates ERK-dependent activation of $\mathrm{p} 21^{\mathrm{CiP} / \mathrm{WAF}}$, and thus inhibits proliferation of colorectal cancer (18). We showed that ERK is activated by S. typhimurium in a time-dependent manner in intestinal epithelial cells with peaks at 40-60 min and at $100 \mathrm{~min}$. Caco-2 and T84 cells show some baseline activation of ERK in uninfected cells. ERK-activation is reduced by L. rhamnosus GG conditioned media in both zinc-deficient and -sufficient Caco-2 cells. However, in zinc-deficient enterocytes, ERK activation is almost totally abolished by probiotic conditioned media, suggesting an increased preventive effect of probiotics in intestinal zinc deficiency. In contrast, purified L. crispatus bacteria are not able to reduce ERK-activation in infected enterocytes. Infected enterocytes also show an increase in phosphorylated $\mathrm{p} 38$, which is inhibited by L. rhamnosus GG conditioned medium. Yan et al. (2002) described that viable $L$. rhamnosus GG inhibits TNF-induced activation of p38 whereas probiotic conditioned media or heat-killed bacteria are not active.

There have been no reports on a combined effect of probiotics and zinc on ERK and p38 activation as part of the MAP kinase pathways. In the future studies with MAP kinase pathway inhibitors will be helpful to determine which and to what degree the specific MAP kinase pathways are involved.

Our observations indicate that both zinc and probiotics have the potential to influence MAP kinase activation enhanced by S. typhimurium and alter enterocyte inflammation. We have shown that probiotic conditioned media enhances the suspension of MAP kinase activity in zinc-deficient infected enterocytes and suggest that the effect of zinc and probiotics may be synergistic.

Several representative probiotic strains were noted to be significantly inhibitory in S. typhimurium. Active inhibitory factor(s) found in the probiotic conditioned media were significantly more inhibiting than purified bacteria. The small inhibitory effect of purified probiotic bacteria might be due to continuous secretion of inhibitory factors or their binding to the probiotic bacterial membrane. These findings suggest that the vehicle of probiotic activity could be used in the future as treatment for intestinal infection.

The inhibitory probiotic effect appears to be primarily mediated by soluble probiotic factors, as probiotic bacteria are significantly less effective in preventing invasion and IL-8 response. We showed that the inhibitory probiotic factors are 
neither a protein nor nucleic acid, are resistant to heat and $\mathrm{pH}$ changes, and work in a dose-dependent manner. Lactic acid has inhibitory properties, but the L. rhamnosus GG 5-10 kD fraction produces substantial additional inhibition. A previous study has shown the secretion of two heat and protease sensitive proteins by L. rhamnosus GG with a molecular size of 80 and $42 \mathrm{kD}$ (19) exhibiting a concentration-dependent activation of anti-apoptotic Akt and inhibition of p38 MAP kinase-induced apoptosis in intestinal epithelial cells. The effects appear to require live bacteria as heat treatment abolishes probiotic effects. Tao et al. (20) describe a low molecular weight peptide from L. rhamnosus GG that activates MAP kinases and induces heat shock proteins (hsp25 and hsp72) in enterocytes. The secreted soluble factor is acid and heat stable and activates MAP kinases (20). However, both studies were conducted in immature young adult mouse colon (YAMC) cells representing immature intestinal cell lines differing from our experiments in mature intestinal epithelial cells. It is highly probable that L. rhamnosus GG secretes more than one active probiotic factor and that the degree of maturity of enterocytes may influence the inflammatory response. The inhibitory factor(s) could either be a polysaccharide or a fatty acid. Elimination or inhibition of function of polysaccharides and fatty acids will determine the nature of the inhibitory factor(s).

As a result, different probiotic bacteria and zinc play a significant role in innate intestinal mucosal immunity. We have shown that probiotics can overcome immune dysfunction of zinc-deficient enterocytes toward enteropathogenic bacteria in a synergistic manner. We speculate that probiotics are specifically active on the enterocyte and not on the microbial flora or the mucosal immune system. Our observations provide a possible explanation for how probiotics reduce the duration and severity of infectious diarrhea in zinc deficiency and the increased susceptibility of zinc-deficient enterocytes toward enteropathogenic bacteria. Ongoing studies are designed to further identify and characterize these probiotic inhibitory factor(s).

In conclusion, a combination of probiotics and zinc might be an inexpensive tool in the prevention and treatment of infectious diarrhea in developing and developed countries. Before establishing a treatment plan for infectious diarrhea our observations have to be confirmed by in vivo studies and double-blind randomized controlled placebo studies. Furthermore, we have to bear in mind that probiotics have strain- specific effects and that different probiotics produce different quantities of a variety of active inhibitory factors.

Acknowledgments. The authors thank Dr. Chitra Amarasiriwardena, Trace-metal Laboratory, Channing Laboratory, Harvard Medical School, Boston, MA, for determining zinc concentrations by ICP-MS (Varian Instruments, Palo Alto, CA).

\section{REFERENCES}

1. Fischer Walker C, Black RE 2004 Zinc and the risk for infectious disease. Annu Rev Nutr 24:255-275

2. Koren-Michowitz M, Dishy V, Zaidenstein R, Yona O, Berman S, Weissgarten J, Golik A 2005 The effect of losartan and losartan/hydrochlorothiazide fixedcombination on magnesium, zinc, and nitric oxide metabolism in hypertensive patients: a prospective open-label study. Am J Hypertens 18:358-363

3. Prasad AS 2001 Recognition of zinc-deficiency syndrome. Nutrition 17:67-69

4. Castillo-Duran C, Vial P, Uauy R 1988 Trace mineral balance during acute diarrhea in infants. J Pediatr 113:452-457

5. Hoque KM, Binder HJ 2006 Zinc in the treatment of acute diarrhea: current status and assessment. Gastroenterology 130:2201-2205

6. Broekaert IJ, Walker WA 2006 Probiotics and chronic disease. J Clin Gastroenterol 40:270-274

7. Shi HN, Walker A 2004 Bacterial colonization and the development of intestinal defences. Can J Gastroenterol 18:493-500

8. Szajewska H, Mrukowicz JZ 2001 Probiotics in the treatment and prevention of acute infectious diarrhea in infants and children: a systematic review of published randomized, double-blind, placebo-controlled trials. J Pediatr Gastroenterol Nutr 33:S17-S25

9. Huang JS, Bousvaros A, Lee JW, Diaz A, Davidson EJ 2002 Efficacy of probiotic use in acute diarrhea in children: a meta-analysis. Dig Dis Sci 47:2625-2634

10. Bao B, Prasad AS, Beck FW, Godmere M 2003 Zinc modulates mRNA levels of cytokines. Am J Physiol Endocrinol Metab 285:E1095-E1102

11. Claud EC, Savidge T, Walker WA 2003 Modulation of human intestinal epithelial cell IL-8 secretion by human milk factors. Pediatr Res 53:419-425

12. Fusunyan RD, Nanthakumar NN, Baldeon ME, Walker WA 2001 Evidence for an innate immune response in the immature human intestine: toll like receptors on fetal enterocytes. Pediatr Res 49:589-593

13. Mengheri E, Nobili F, Vignolini F, Pesenti M, Brandi G, Biavati B 1999 Bifidobacterium animalis protects intestine from damage induced by zinc deficiency in rats. J Nutr 129:2251-2257

14. Shamir R, Makhoul IR, Etzioni A, Shehadeh N 2005 Evaluation of a diet containing probiotics and zinc for the treatment of mild diarrheal illness in children younger than one year of age. J Am Coll Nutr 24:370-375

15. Huang FC, Werne A, Li Q, Galyov EE, Walker WA, Cherayil BJ 2004 Cooperative Interactions between Flagellin and SopE2 in the epithelial interleukin-8 response to Salmonella enterica Serovar Typhimurium infection. Infect Immun 72:5052-5062

16. Nobili F, Vignolini F, Figus E, Mengheri E 1997 Treatment of rats with dexamethasone and thyroxine protects against zinc deficiency-induced intestinal damage. J Nutr 127:1807-1813

17. Azriel-Tamir H, Sharir H, Schwartz B, Hershfinkel M 2004 Extracellular zinc triggers ERK-dependent activation of $\mathrm{Na}^{+} / \mathrm{H}^{+}$exchange in colonocytes mediated by the zinc-sensing receptor. J Biol Chem 279:51804-51816

18. Park KS, Ahn Y, Kim JA, Yun MS, Seong BL, Choi KY 2002 Extracellular zinc stimulates ERK-dependent activation of $\mathrm{p} 21^{\mathrm{Cip} / \mathrm{WAF} 1}$ and inhibits proliferation of colorectal cancer cells. Br J Pharmacol 137:597-607

19. Yan F, Polk DB 2002 Probiotic bacterium prevents cytokine-induced apoptosis in intestinal epithelial cells. J Biol Chem 277:50959-50965

20. Tao Y, Drabik KA, Waypa TS, Musch MW, Alverdy JC, Schneewind O, Chang EB, Petrof EO 2006 Soluble factors from Lactobacillus $G G$ activate MAPKs and induce cytoprotective heat shock proteins in intestinal epithelial cells. Am J Physiol Cell Physiol 290:C1018-C1030 